\title{
Business model: Desvendando o construto
}

\author{
Cyntia Vilasboas Calixto ${ }^{1}$ e Maria Tereza Leme Fleury \\ Fundação Getúlio Vargas - EAESP/FGV, São Paulo/SP, Brasil
}

\section{DETALHES DO ARTIGO}

\section{Histórico do artigo:}

Recebido em 31 de Outubro de 2014

Aceito em 12 de Junho de 2015

Disponível online em 30 de Agosto de 2015

Sistema de Revisão "Double Blind Review"

\section{Editores científicos}

Gabriel Vouga Chueke

Marcos Amatucci

\section{Palavras-chaves:}

Business model

Empresas Multinacionais

Negócios Internacionais

\author{
RESUMO
}

O presente estudo realiza uma revisão sistemática da literatura a fim de identificar as principais definições de business model (modelo de negócio), bem como os elementos que compõem este construto. A amostra final analisada são 81 artigos publicados em periódicos internacionais com fator de impacto superior a 1,5, segundo os critérios do Journal Citation Report (JCR). Percebeuse que a relação entre business model e empresas multinacionais vem sido tangenciada pelos pesquisadores e, portanto, apareceu como oportunidade de investigação. Considerando que os business models descrevem como uma empresa cria valor por meio da combinação de recursos externos e internos em seu conjunto de atividades, é relevante compreender os elementos de seu design para a determinação do modelo de negócios da empresa multinacional.

(C) 2015 Internext | ESPM. Todos os direitos reservados!

\section{Introdução}

Business models, ou modelo de negócios, é um conceito que vem sido estudado de forma recorrente nos últimos anos na área de gestão. Embora tenha recebido atenção de acadêmicos e consultores, não existe uma definição aceita como consensual. Isto se deve às diferentes lentes teóricas utilizadas pelos autores (BADEN-FULLER; MORGAN, 2010). Duas edições do periódico Long Range Planning sobre business models (em 2010 e 2013) e uma especial do Strategic Organization (2013) ampliaram a discussão sobre o tema e contribuiu para o desenvolvimento de pesquisas posteriores.

Revisões da literatura sobre business models (KLANG; WALLNOFER; HACKLIN, 2014; ZOTT, AMIT; MASSA, 2011) indicam que o interesse no tema remete ao início da década de 90, em função da internet, do rápido crescimento dos mercados emergentes e da expansão comercial. Esses elementos alteraram as formas tradicionais de criar e entregar valor, proporcionando o desenvolvimento de novas configurações organizacionais e adaptações à inovação (ZOTT et al, 2011). Há uma mudança no

\footnotetext{
${ }^{1}$ Contato da autora: Email: cyntiacalixto@gmail.com
}

paradigma de fazer negócios da Era Industrial para a Era da Informação (DASILVA; TRKMAN, 2013). Logo, tendo em vista que gestão é dependente do contexto, novos business models foram (re)criados para atender esses desafios, em especial pelas empresas multinacionais.

Uma grande parte dos estudos acerca de Business Models é de caráter teórico, definindo o construto ou sua apresentando a sua evolução (SHAFER; SMITH; LINDER, 2005; BADEN-FULLER; MORGAN, 2010; TEECE, 2010; ZOTT; AMIT, 2010; ZOTT et al, 2011; DASILVA; TRKMAN, 2013; BADEN-FULLER; HAEFLIGER, 2013; KLANG et al, 2014); pesquisas empíricas são majoritariamente estudos de caso, que avaliam ou descrevem o modelo de negócios de uma ou mais empresas (SANTOS, SPECTOR, VAN DER HEYDEN; 2009; DEMIL; LECOQC, 2010; WIRTZ, SCHILKE; ULLRICH, 2010; SMITH, BINNS; TUSHMAN, 2010; ASPARA, LAMBERG; LAUKIA; TIKKANEN, 2013). Dos poucos estudos quantitativos publicados $e$ avaliados nesta pesquisa, destaca-se o uso de surveys associando Business Models com desempenho.

(C) 2015 Internext | ESPM. Todos os direitos reservados! Doi: dx.doi.org/10.18568/1980-486510218-302015 
Este estudo difere-se das meta-analises, revisões e bibliometrias previamente realizadas sobre o tema, uma vez que além de analisar o histórico e evolução do conceito de business model, também buscou verificar a relação com empresas multinacionais nestes estudos. Para isso, avaliou-se em todos os estudos selecionados quando este tipo de organização fez parte da amostra ou da compreensão do conceito.

Sendo assim, identificou-se que no contexto de estratégia internacional, poucos estudos envolveram empresas multinacionais (MNE). Recentemente, o artigo de cunho teórico de Tallman (2014) propõe que é necessário adaptar o business model aos locais de operação das atividades da multinacional e propõe a noção de Global Business Model. Quanto aos estudos empíricos já publicados, as multinacionais apareceram como objeto de análise de estudos de caso ou como contextualização do que seria um business model, enfatizando a complexidade da estrutura organizacional.

Destaca-se que nenhum dos artigos avaliados nesta pesquisa, por meio da revisão sistemática da literatura, abordou a relação dos business models entre matriz e subsidiária de empresas multinacionais. Considerando que os business models descrevem como uma empresa cria valor por meio da combinação de recursos externos e internos em seu conjunto de atividades (SANTOS et al, 2009; ZOTT et al, 2011), acredita-se que seja relevante compreender os elementos de seu design para a determinação do modelo de negócios de uma empresa multinacional.

Portanto, para captar a essência do design do modelo de negócio de empresas multinacionais, o presente estudo busca compreender inicialmente com base na literatura: Quais são os elementos que compõem um business model?

Para responder esse questionamento, a pesquisa foi ancorada nas técnicas de revisão sistemática da literatura e realizou-se uma análise em profundidade dos artigos publicados em periódicos internacionais.

Este artigo está dividido em cinco seções. Após a introdução, apresentam-se na revisão da literatura as principais definições de business model, bem como sua evolução e a relação com negócios internacionais. Posteriormente, explicita-se a metodologia utilizada para a realização do estudo e os principais resultados. Por fim, considerações finais acerca da pesquisa e indicações de estudos futuros sobre a temática em questão.

\section{Revisão da literatura}

Nesta seção, apresenta-se uma síntese dos temas abordados para a construção teórica deste estudo. 0 intuito principal foi analisar a evolução do tema e identificar os principais elementos do business model abordados nos estudos internacionais já publicados.

\subsection{Business Model: O Construto}

Embora não exista uma aceitação consensual acerca da definição de business models, alguns autores tornaram-se referência na área tendo a sua perspectiva e sua proposta do construto utilizada em estudos posteriores. Dos artigos analisados nesta revisão, pode-se destacar como as definições mais utilizadas entre os autores foram aquelas desenvolvidas por Amit \& Zott (2001) e Teece (2010). A maioria dos estudos derivou suas próprias

Tab. 1

Principais definições de Business Model

\begin{tabular}{|c|c|c|}
\hline Autor & Definição de Business Model & Artigos que usaram essas definições \\
\hline $\begin{array}{l}\text { Amit \& Zott } \\
\text { (2001) }\end{array}$ & $\begin{array}{l}\text { "Um modelo de negócios descreve o design do conteúdo, da estrutura } \\
\text { e da governança de uma transação para criar valor por meio da } \\
\text { exploração das oportunidades de negócio "(p. 493). }\end{array}$ & $\begin{array}{l}\text { Zott \& Amit (2007), Zott \& Amit } \\
\text { (2008), Santos et al (2009), Sosna et } \\
\text { al (2010), Bock et al (2010) and } \\
\text { Markides (2013). }\end{array}$ \\
\hline Teece (2010) & $\begin{array}{l}\text { "descreve o design ou arquitetura da criação de valor, entrega e } \\
\text { mecanismos de captura que são por ele empregados. A essência do } \\
\text { modelo de negócios é definir a maneira que a empresa entrega valor } \\
\text { aos seus clientes, atrai os clientes para pagar pelo valor e converte } \\
\text { esses pagamentos em lucro". (p. 172) }\end{array}$ & $\begin{array}{l}\text { Baden-Fuller \& Morgan (2010), } \\
\text { Dunford et al (2010), Gambardella \& } \\
\text { McGahan (2010) Bock et al (2012), } \\
\text { Runfola et al (2013), Eckhardt (2013) }\end{array}$ \\
\hline
\end{tabular}

Fonte: As autoras

Internext | São Paulo, v.10, n. 2, p. 18-30, mai./ago. 2015 
definições com base nelas. Assim, inicia-se este subcapítulo apresentando-as, conforme indicado na tabela 1.

Notou-se que a maioria dos estudos buscava descrever o que seria um business model, geralmente relacionando o seu design (ou estrutura) com à criação de valor, ancorados nos textos de Amit \& Zott (2001) e, posteriormente, Teece (2010). Contudo, na análise dos textos perceberam-se diferenças na forma em que os autores tratavam o construto business model.

Zott, Amit e Massa (2011) relataram algumas definições de business model que encontraram ao longo da sua síntese da literatura. Assim, este estudo partiu da classificação já estabelecida pelos autores e avaliaram-se os estudos nesta revisão sistemática em: declaração, descrição, representação, arquitetura, modelo conceitual, modelo estrutural, método, framework ou padrão. Da mesma forma do que foi mencionado pelos autores, é surpreendente o número de artigos que não apresenta de maneira explícita o conceito a ser considerado ao longo do estudo (22\%), realizando apenas citações de diversos estudos anteriores.

Com base na classificação de Zott et al. (2011), analisaram-se os artigos com a temática de business model (detalhes da seleção estão disponíveis no capítulo de metodologia). Salienta-se que os mesmos não expressaram características de declaração ou padrão. Tendo em vista que há uma linha tênue entre termos descrição e representação, optou-se por unir os conteúdos em uma única classificação. Dessa forma, os artigos que compuseram a análise apresentaram as características indicadas na tabela 2 .

Como se pode perceber pelos resultados do quadro 2, os estudos de Amit \& Zott (2001) e Teece (2010) foram relevantes para o desenvolvimento da literatura do construto, influenciando significativamente na forma em que ele é interpretado. Essa tendência acaba sendo refletida nos componentes do construto, que tem como elemento central a criação de valor e a captação de valor.

Nesta pesquisa, compreende-se, com base nos estudos de Amit e Zott (2001) e Zott et al (2011), que Business Model é composto por um conjunto de atividades interdependentes além das fronteiras da firma, que descreve o conteúdo, a estrutura e a governança a fim de criar valor à organização.

\subsection{Evolução dos estudos sobre Business Model}

Business Model faz parte da literatura em gestão e negócios há bastante tempo (BADEN-FULLER; MANGEMATIN, 2013). Santos et al. (2009) indicam que o primeiro texto abordando o construto, mesmo que indiretamente seja datado em 1947. As revisões recentes da literatura (KLANG; WALLNOFER; HACKLIN, 2014; ZOTT, AMIT; MASSA, 2011) indicam que o interesse crescente sobre o tema durante a década de 90 seja oriundo da revolução da internet e seus impactos nas transações comerciais, do rápido

Tab. 2

Forma de análise do conceito

\begin{tabular}{|c|c|c|}
\hline Classificação & Definição Operacional & $\begin{array}{l}\text { Número de } \\
\text { estudos }\end{array}$ \\
\hline Descrição/ Representação & $\begin{array}{l}\text { Quando havia a descrição/representação da forma de fazer negócios de } \\
\text { uma empresa. }\end{array}$ & 20 \\
\hline Arquitetura & $\begin{array}{l}\text { Utilizado quando o termo arquitetura era indicado pelos próprios autores, } \\
\text { como Teece (2010). }\end{array}$ & 12 \\
\hline Modelo Conceitual & $\begin{array}{l}\text { Utilizado quando termo conceitual era mencionado nos estudos pelos } \\
\text { próprios autores, como Mutka \& Aaltonen (2013). }\end{array}$ & 2 \\
\hline Modelo Estrutural & $\begin{array}{l}\text { Zott et al (2011) classificaram seus estudos como modelo estrutural. Assim, } \\
\text { todos aqueles que usavam a mesma definição ou envolviam sistema de } \\
\text { atividades, foram classificados nesta categoria. }\end{array}$ & 27 \\
\hline Framework & $\begin{array}{l}\text { Utilizado quando os autores realizavam frameworks de business model ou } \\
\text { indicavam o termo nos seus estudos. }\end{array}$ & 12 \\
\hline Não foi possível classificar & & 8 \\
\hline
\end{tabular}

Fonte: As autoras 
crescimento dos mercados emergentes e da expansão comercial que ampliou a concorrência internacional.

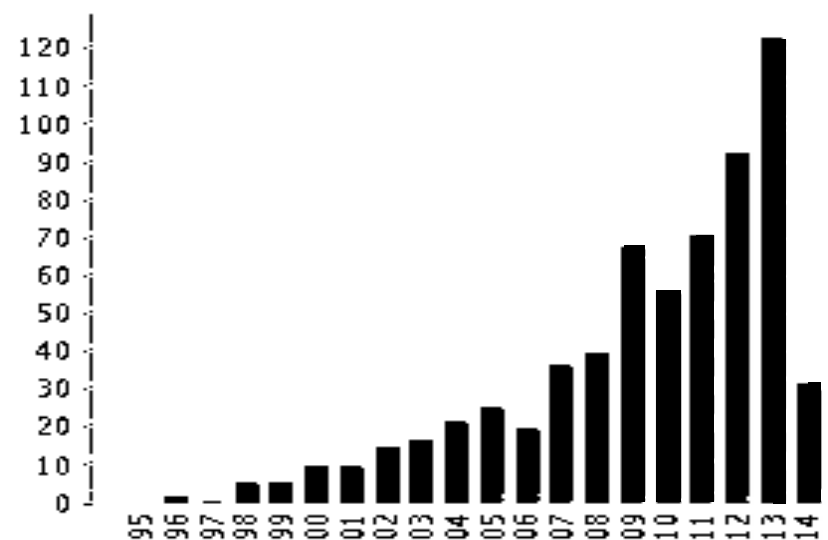

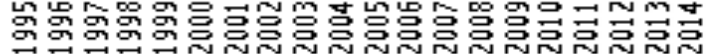

Figura 1: Artigos publicados por ano com o tópico "business model"

Fonte: Adaptado dos resultados da pesquisa no ISI Web of knowledge em 07/06/2014.

Percebe-se pela figura 1 o acréscimo da discussão sobre business models após 2009, corroborando com as edições especiais do Long Range Planning $e$ Strategic Organization. Além disso, embora possua um caráter aplicado, o livro Business Model Generation, com o famoso modelo Canvas de Alexander Osterwalder e Yves Pigneur, foi lançado em 2010, repercutindo significativamente para a divulgação do tema e atraindo também o interesse dos acadêmicos.

Considerando que ocorreram mudanças nas formas tradicionais de criar e entregar valor, desenvolveram-se novas configurações organizacionais e adaptações à inovação, com destaque os modelos de negócio das empresas para atender esse novo contexto (ZOTT et al, 2011).

Uma grande parte dos estudos avaliados nesta revisão sobre a temática de business model são de caráter teórico, definindo o construto ou sua apresentando a sua evolução (SHAFER; SMITH; LINDER, 2005; BADEN-FULLER; MORGAN, 2010; TEECE, 2010; ZOTT; AMIT, 2010; ZOTT et al, 2011; DASILVA; TRKMAN, 2013; BADEN-FULLER; HAEFLIGER, 2013; KLANG et al, 2014). Contudo, percebeu-se a necessidade de realizar estudos empíricos a fim de legitimar o termo e as teorias que o embasam, além de testar as teorias sobre como a empresa entrega valor aos seus clientes (ECKHARDT, 2013). Até o momento, dominam os estudos de caso, onde há descrição do business model de empresas selecionadas.

Ainda que os termos Business Model e Business Strategy apareçam de forma intercambiável em alguns artigos, eles não representam o mesmo construto. No Business Strategy especifica-se o que a empresa oferece (what), quem são os seus clientes (who) e como produzir e entregar valor aos seus clientes (how). A relação do Business Model com Business Strategy está no como entregar valor aos clientes (SANTOS; SPECTOR; VAN DER HEYDEN, 2009; CASADESUS-MASANELL; RICART, 2010).

Zott et al. (2011) indicam três grandes áreas de estudo sobre business model: e-business, business model innovation e estratégia. Sinteticamente, os

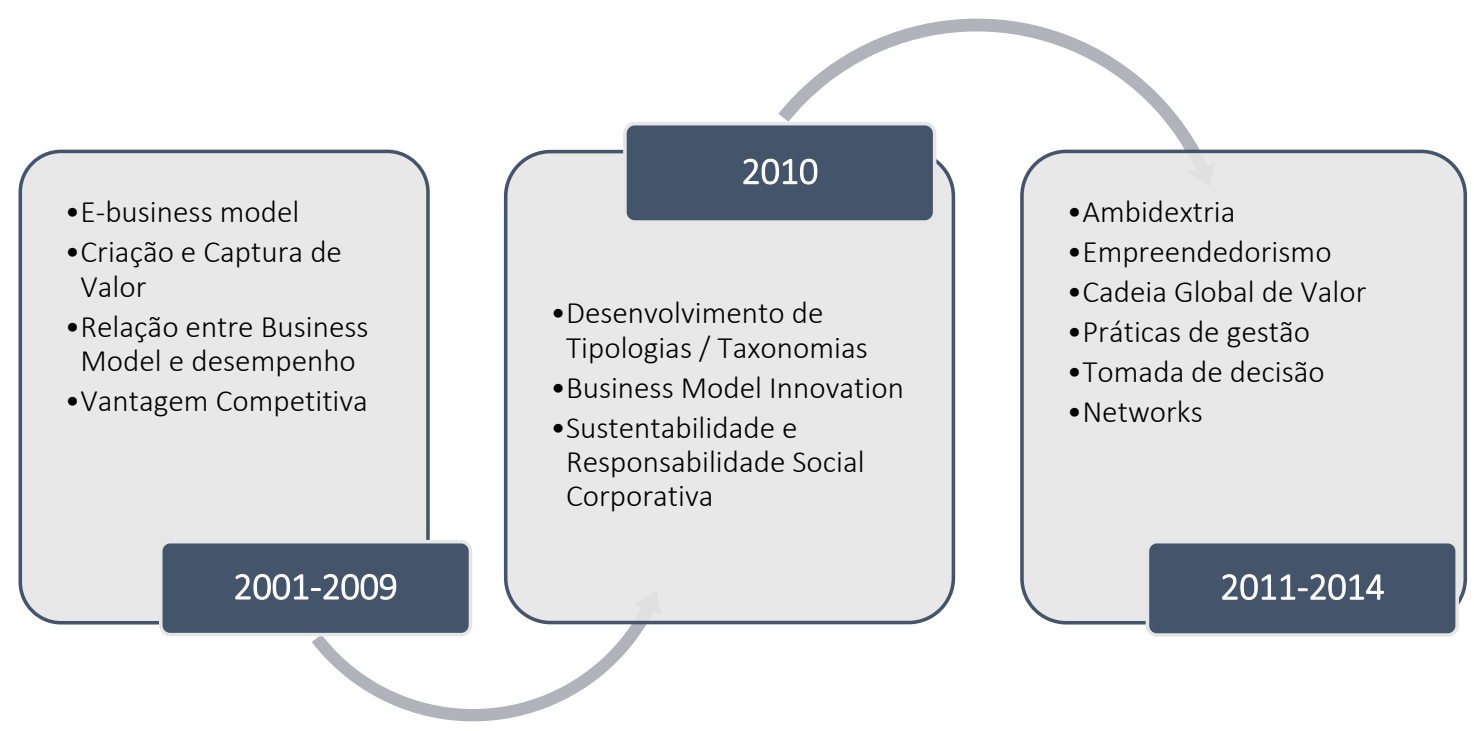

Figura 2: Evolução da abordagem sobre Business Models Fonte: As Autoras 
estudos envolvendo e-business envolvem a necessidade de representação genérica de ebusiness models (MAHADEVAN, 2000; AMIT; ZOTT, 2001) e o desenvolvimento de tipologias/taxonomias (WIRTZ et al, 2010).

As publicações acerca de Business Model Innovation abrangem o business model como um mecanismo capaz de conectar a tecnologia das empresas com as necessidades de seus clientes ou outros recursos da própria organização (CAGNINA; POIAN, 2009; CHESBROUGH, 2010; GAMBARDELLA; MCGAHAN, 2010; ZOTT et al, 2011). Ainda, a capacidade e os desafios da empresa em lidar com dois business models concomitantemente (características de ambidextria) (MARKIDES, 2013; MCNAMARA et al, 2013), reconfiguração das atividades existentes (WILLEMSTEIN et al, 2007; SOSNA et al, 2010; BOCK et al, 2012; WITELL; LOGREN, 2013), empreendedorismo (DOGANOVA; EYQUEM-RENAULT, 2009; TRIMI; BERBEGALMIRABENT, 2012) e desenvolvimento de novas proposições de valor à organização (DAHAN et al, 2010; YUNUS et al, 2010).

Os estudos relacionados com estratégia empresarial estão dirigidos na forma que as empresas criam valor, no desempenho e na vantagem competitiva. Amit e Zott (2001) postulam quatro as fontes de criação de valor por meio de business models: novelty (focado na inovação de Schumpeter), lock-in (relacionado às networks estratégicas), complementarities (ancorado na literatura de Resource-Based View - RBV) e efficiency (com foco na literatura de Transaction Cost Economics - TCE).

Em relação ao desempenho, os estudos variam desde o teste do business model apropriado para gerar uma melhor performance para a organização (ZOTT; AMIT, 2013; ZOTT; AMIT, 2008; HUELSBECK et al, 2011) até o uso do business model como uma variável moderadora para a relação da alta gestão com a performance da empresa (PATZELT et al, 2008). Já as publicações acerca de vantagem competitiva, relacionam o construto com sustentabilidade (BRYSON; LOMBARDI, 2009; GIROTRA; NETESSINE, 2013; BENIJTS, 2014), capacidades e práticas de gestão (STORBACKA, 2011) e aprendizagem (ITAMI; NISHINO, 2010).

Embora existam autores que não aceitem business model como uma teoria (AREND, 2013), pois creem que exista a sobreposição das ideias com outros conceitos já estabelecidos, acredita-se que já exista validade suficiente do construto e sua contribuição teórica. A sobreposição com outros construtos vem sido dirimidas nos estudos recentes, quando os pesquisadores já possuem mais clareza do que compõe um business model e o articulam com propriedade sobre o sua base de estudo (ex: arquitetura, sistema de atividades e etc...) (ZOTT; AMIT, 2013).

\subsection{Business Models e Negócios Internacionais}

Embora a revisão sistemática da literatura tenha focado na presença ou relevância de empresas multinacionais sobre o tema, poucos estudos avaliaram a questão do business model e corporações multinacionais. A grande maioria deles utilizou como objeto de análise uma empresa multinacional para seu estudo de caso ou contextualização do que seria um business model (PATZELT et al., 2008; CASADESUS-MASANELL; RICART, 2010; DAHAN et al., 2010; DUNFORD et al., 2010; GUERCINI; RUNFOLA et al , 2012; SABATIER et al., 2010; SOSNA et al., 2010; STORBACKA, 2011; BOCK et al., 2012; ASPARA et al., 2013). Entretanto, esses estudos não consideraram o seu contexto de atuação internacional da empresa como eixo de análise, focando na complexidade organizacional da mesma.

Tallman (2014), num artigo apresentado recentemente, que não fez parte dessa revisão sistemática da literatura, foi o único autor identificado até o momento que abordou este tema. De acordo com o autor, as publicações vigentes sobre business model não reconhecem que diferentes mercados podem demandar estruturas organizacionais distintas. Assim, ele postula que o valor dos recursos e das capacidades varia conforme o país de destino e estratégia da empresa deve ajustar conforme as condições do mercado. Ainda, as estruturas organizacionais da empresa multinacional variam conforme as restrições regulatórias, instabilidade política, desenvolvimento dos mercados e as capacidades técnicas dos seus fornecedores (TALLMAN, 2014).

Portanto, para Tallman (2014) uma empresa multinacional precisa adaptar seus business models às diferentes condições dos locais onde possuem operações. A criação de valor estaria ligada aos firmspecific assets (FSA) da organização, já a entrega de valor relaciona-se com as estruturas organizacionais 
da multinacional, ou seja, como as atividades são realizadas, por quem e como são coordenadas (cadeia de valor) e são adaptáveis conforme a eficiência da relação empresa-cliente. Por fim, a captura de valor estaria relacionada à habilidade da organização em acessar os ganhos que foram coletados por suas operações internacionais.

Contudo, ressalta-se que o estudo de Tallman está limitado à proposição teórica de um Global Business Model, não apresentando também uma aplicação empírica do business model de uma empresa multinacional.

\section{Metodologia}

Nesta revisão sistemática examinaram-se os artigos publicados em periódicos internacionais, procurando identificar padrões e relações entre os autores acerca do construto business model. Considerando que o objetivo central da pesquisa foi desvendar os elementos que compõem um business model, optouse em utilizar a técnica de revisão sistemática da literatura para descobrir quais foram os principais componentes citados pelos estudos anteriores e não desenvolver novas tipologias. Por meio de agrupamento por afinidade conceitual foi possível reunir as categorias citadas e desenvolver uma análise descritiva do construto.

Para conduzir a revisão sistemática da literatura, utilizou-se o método indicado por Arlene Fink (2010), que é composto por diversas etapas. Segundo a autora, por meio da revisão sistemática é possível identificar, avaliar e sintetizar o que já foi discorrido pelos pesquisadores do tema. Assim, o processo foi dividido em dividido em sete etapas:

- Seleção de uma pergunta de pesquisa que norteia o estudo;

- Seleção de uma base de dados eletrônica;

- Escolha dos termos de busca;

- Aplicação de critérios de seleção do material (idioma, conteúdo, forma);

- Aplicação dos critérios metodológicos de seleção (adequação e qualidade);

- Revisão;

- Sintetização dos resultados (descritiva ou meta-análise) (FINK, 2010).

Este estudo usou todos os critérios indicados por Fink (2010) para sua execução, iniciando a busca de artigos que de alguma forma explicassem o que é um business model?. Optou-se por uma pergunta bastante ampla, para verificar de que forma o conceito está sendo utilizado na literatura.

A base de dados de artigos selecionada para a pesquisa foi a Web of Science (ISI Web of Knowledge) em função de ser uma base multidisciplinar e que indexa os periódicos mais renomados de cada área. A pesquisa foi conduzida em junho de 2014, cobriu o período de busca de 1945-2014, pois este é o ciclo disponível na plataforma, e conteve o termo "business model" como tópico (entre aspas para que as palavras aparecessem sempre juntas, técnica de codificação booleana). Como resultado inicial, apareceram 5.261 arquivos. A utilização dos citation reports (planilhas compostas pelos artigos resultantes da pesquisa na base) é relevante em função do mapeamento disponível dos artigos publicados pelos critérios de busca. Além disso, proporciona uma visão gráfica da evolução e tendências de pesquisa no período em questão. (KEAR; COLBERT-LEWIS, 2011).

Após a seleção do domínio de pesquisa social science $(n=1.489)$, das áreas de pesquisa business economics e operations research management science $(n=1.251)$, do tipo de documento article ( $n=669)$ e dos idiomas English, Portuguese e Spanish (que são os idiomas de domínio das pesquisadoras), permaneceram 657 artigos para análise. Elegeram-se somente artigos na revisão, pois eles estão sujeitos a revisão as cegas (blind review), ou seja, o conhecimento já foi previamente validado (KLANG et al, 2014).

Para assegurar a qualidade do material utilizado, optou-se por usar a listagem do Journal Citation Report (JCR) e selecionar os 100 periódicos com maior fator de impacto (acima de 1,5 ) nas áreas de business, business \& finance e management. O JCR é uma métrica reconhecida internacionalmente com o propósito de comparar as citações de artigos publicados entre periódicos (THOMPSON REUTERS, 2008) e pode ser interpretado como um indicador de sucesso da publicação em periódicos renomados. A escolha de artigos publicados em periódicos com fator de impacto superior a 1,5 ocorreu para assegurar o uso de periódicos maduros e com credibilidade internacional. Assim, todos os artigos que foram publicados em periódicos listados ficariam na amostra para serem revisados $(n=246)$.

A revisão iniciou pela análise dos resumos (abstracts). Desconsideraram-se artigos que desenvolveram business model de forma tangencial, 
ou seja, o construto não aparecia na análise nem na fundamentação teórica. Dessa forma, identificaramse 101 artigos como relevantes para a pesquisa. A
Os textos foram organizados com o auxílio do software Atlas Ti. Os documentos foram lidos e codificados nesta plataforma. Inicialmente,

Tab. 3

10 artigos mais citados sobre Business Model - amostra final

\begin{tabular}{|c|c|c|c|c|c|c|c|}
\hline Título & Autores & Periódico & $\begin{array}{c}\text { Ano da } \\
\text { publicação }\end{array}$ & $\begin{array}{l}\text { Total de } \\
\text { citações }\end{array}$ & Objetivo da pesquisa & Amostra & $\begin{array}{l}\text { Técnica de coleta e } \\
\text { análise de dados }\end{array}$ \\
\hline $\begin{array}{l}\text { Value creation in e- } \\
\text { business }\end{array}$ & $\begin{array}{l}\text { Amit, R; } \\
\text { Zott, C }\end{array}$ & $\begin{array}{l}\text { Strategic } \\
\text { Management } \\
\text { Journal }\end{array}$ & 2001 & 592 & $\begin{array}{l}\text { Identify value creation } \\
\text { sources in e-business }\end{array}$ & $\begin{array}{l}59 \text { e-business } \\
\text { enterprises }\end{array}$ & $\begin{array}{l}\text { Multiple Case Studies } \\
\text { (Interviews with Semi- } \\
\text { structured } \\
\text { questionnaires) }\end{array}$ \\
\hline $\begin{array}{l}\text { Replication as } \\
\text { strategy }\end{array}$ & $\begin{array}{l}\text { Winter, SG; } \\
\text { Szulanski, G }\end{array}$ & $\begin{array}{l}\text { Organization } \\
\text { Science }\end{array}$ & 2001 & 232 & $\begin{array}{l}\text { Present the main elements of } \\
\text { replication strategy }\end{array}$ & 1 bank & $\begin{array}{l}\text { Case Study } \\
\text { (Illustrative) }\end{array}$ \\
\hline $\begin{array}{l}\text { Why business } \\
\text { models matter }\end{array}$ & Magretta, J & $\begin{array}{l}\text { Harvard } \\
\text { Business } \\
\text { Review }\end{array}$ & 2002 & 191 & $\begin{array}{l}\text { Clarify what a business model } \\
\text { is and its relation to strategy }\end{array}$ & Non-applicable & \\
\hline $\begin{array}{l}\text { Business Models, } \\
\text { Business Strategy } \\
\text { and Innovation }\end{array}$ & $\begin{array}{l}\text { Teece, David } \\
\text { J. }\end{array}$ & $\begin{array}{l}\text { Long Range } \\
\text { Planning }\end{array}$ & 2010 & 173 & $\begin{array}{l}\text { Understand the relevance of } \\
\text { the business model and } \\
\text { explore the connections to } \\
\text { strategy, innovation, and } \\
\text { economic theory }\end{array}$ & Non-applicable & \\
\hline $\begin{array}{l}\text { Business models for } \\
\text { Internet-based E- } \\
\text { commerce: An } \\
\text { anatomy }\end{array}$ & $\begin{array}{l}\text { Mahadevan, } \\
\text { B }\end{array}$ & $\begin{array}{l}\text { California } \\
\text { Management } \\
\text { Review }\end{array}$ & 2000 & 142 & $\begin{array}{l}\text { Develop a framework to } \\
\text { understand the business } \\
\text { model in the on-line context. }\end{array}$ & Non-applicable & \\
\hline $\begin{array}{l}\text { The fit between } \\
\text { product market } \\
\text { strategy and } \\
\text { business model: } \\
\text { Implications for firm } \\
\text { performance }\end{array}$ & $\begin{array}{l}\text { Zott, } \\
\text { Christoph; } \\
\text { Amit, R. }\end{array}$ & $\begin{array}{l}\text { Strategic } \\
\text { Management } \\
\text { Journal }\end{array}$ & 2008 & 121 & $\begin{array}{l}\text { Analyze the fit of the product } \\
\text { strategy with the business } \\
\text { model and its effect for firm } \\
\text { performance. }\end{array}$ & $\begin{array}{l}170 \text { publicly } \\
\text { listed companies }\end{array}$ & $\begin{array}{l}\text { Survey. Multiple } \\
\text { regression } \\
\text { econometric model } \\
\text { (OLS and PLS). }\end{array}$ \\
\hline $\begin{array}{l}\text { Business Model } \\
\text { Innovation: } \\
\text { Opportunities and } \\
\text { Barriers }\end{array}$ & $\begin{array}{l}\text { Chesbrough, } \\
\mathrm{H} \text {. }\end{array}$ & $\begin{array}{l}\text { Long Range } \\
\text { Planning }\end{array}$ & 2010 & 102 & $\begin{array}{l}\text { Explore the barriers of } \\
\text { Business Model Innovation }\end{array}$ & Non-applicable & \\
\hline $\begin{array}{l}\text { Business model } \\
\text { design and the } \\
\text { performance of } \\
\text { entrepreneurial } \\
\text { firms }\end{array}$ & $\begin{array}{l}\text { Zott, C.; Amit, } \\
\text { R. }\end{array}$ & $\begin{array}{l}\text { Organization } \\
\text { Science }\end{array}$ & 2007 & 93 & $\begin{array}{l}\text { Evaluate the impact of the } \\
\text { business model design on the } \\
\text { performance of } \\
\text { entrepreneurial firms. }\end{array}$ & 190 enterprises & $\begin{array}{l}\text { Survey. Multiple } \\
\text { regression (OLS). }\end{array}$ \\
\hline $\begin{array}{l}\text { Organizing for } \\
\text { solutions: Systems } \\
\text { seller vs. systems } \\
\text { integrator }\end{array}$ & $\begin{array}{l}\text { Davies, A.; } \\
\text { Brady, T.; } \\
\text { Hobday, M. }\end{array}$ & $\begin{array}{l}\text { Industrial } \\
\text { Marketing } \\
\text { Management }\end{array}$ & 2007 & 89 & $\begin{array}{l}\text { Investigate foe enterprises } \\
\text { organize internal and } \\
\text { external activities to promote } \\
\text { integrated solutions }\end{array}$ & $\begin{array}{l}5 \text { companies } \\
\text { from different } \\
\text { sectors }\end{array}$ & Multiple Case Study \\
\hline $\begin{array}{l}\text { Entry strategies } \\
\text { under competing } \\
\text { standards: Hybrid } \\
\text { business models in } \\
\text { the open source } \\
\text { software industry }\end{array}$ & $\begin{array}{l}\text { Bonaccorsi, } \\
\text { Andrea; } \\
\text { Giannangeli, } \\
\text { S.; Rossi, C. }\end{array}$ & $\begin{array}{l}\text { Management } \\
\text { Science }\end{array}$ & 2006 & 80 & $\begin{array}{l}\text { Identify the business models } \\
\text { used to enter the open } \\
\text { source movement }\end{array}$ & $\begin{array}{l}146 \text { Italian } \\
\text { software firms }\end{array}$ & Survey. \\
\hline
\end{tabular}

Fonte: As autoras, segundo os dados da ISI Web of Knowledge.

leitura em profundidade dos artigos acabou excluindo mais 20 estudos, por não apresentarem o construto como elemento central, apenas mencionado de forma esporádica ao longo do texto. Logo, a amostra final analisada é de 81 artigos. buscaram-se as definições de business model em cada texto, bem como os componentes que cada autor indicou fazer parte deste construto. Para a análise das definições, classificaram-se os textos conforme as categorias indicadas por Zott et al (2011) e previamente apresentadas no capítulo 2.1. 
Depois, avaliaram-se os artigos mais citados, seus objetivos de pesquisa e métodos utilizados. Além disso, quem são principais autores do tema. Por fim, categorizaram-se os principais componentes de business model para compreender a formação do construto. A seguir, destacam-se alguns resultados da revisão da literatura que concluíram ao atendimento da questão central de pesquisa deste estudo.

\section{Resultados}

Em termos de agregação, esta pesquisa foi realizada em nível micro, ou seja, os artigos resultantes da seleção foram avaliados por indivíduo ou parceria entre dois ou mais autores (GLANZEL, 2003). De acordo com Glazel (2003), alguns fatores podem influenciar as publicações: assunto, idade do autor, seu status social e o período de observação. Embora a pesquisa tenha permitido a busca de artigos a partir de 1945, os estudos apareceram majoritariamente após os anos 2000, o que reduz significativamente o período de análise para 14 anos.

Embora as métricas de indicadores de citação sejam controversas, a análise dos artigos mais citados é relevante para compreender a informação cientifica existente e documentada (GLANZEL, 2003). Assim, o número de citações de um artigo pode ser interpretado como a aceitabilidade daquele estudo pela comunidade acadêmica.

Dentre os artigos mais citados na primeira seleção ( $n=657$ ), momento em que só foram usados os filtros de domínio e área de pesquisa, percebeu-se que havia uma diversidade de periódicos e temáticas de estudo relacionadas ao termo "business model", embora as áreas de pesquisa tenham sido reduzidas na pesquisa. Esta pode ser uma das razões do pouco embate teórico e a dificuldade da formação do conhecimento científico sobre o tema. Notou-se também que autores renomados em outras lentes teóricas como David Teece (capacidades dinâmicas) e Sidney Winter (rotinas) já relacionam o construto com suas temáticas de pesquisa.

Ao final da seleção dos artigos, após a leitura dos resumos e a respectiva adequação com o objetivo da pesquisa ( $n=246)$, apenas quatro artigos dos identificados na primeira seleção permaneceram na lista. Percebe-se que Raphael Amit e Chistoph Zott aparecem como destaque na área, com três artigos listados entre os 10 mais citados (Veja Tabela 3).

Em relação às coautorias, ressalta-se a parceria entre Amit \& Zott com seis publicações sobre o tema, seguidos de Casadesus-Masanell \& Ricart, Sabatier \& Mangematin, George \& Bock, Runfola \& Guercini,
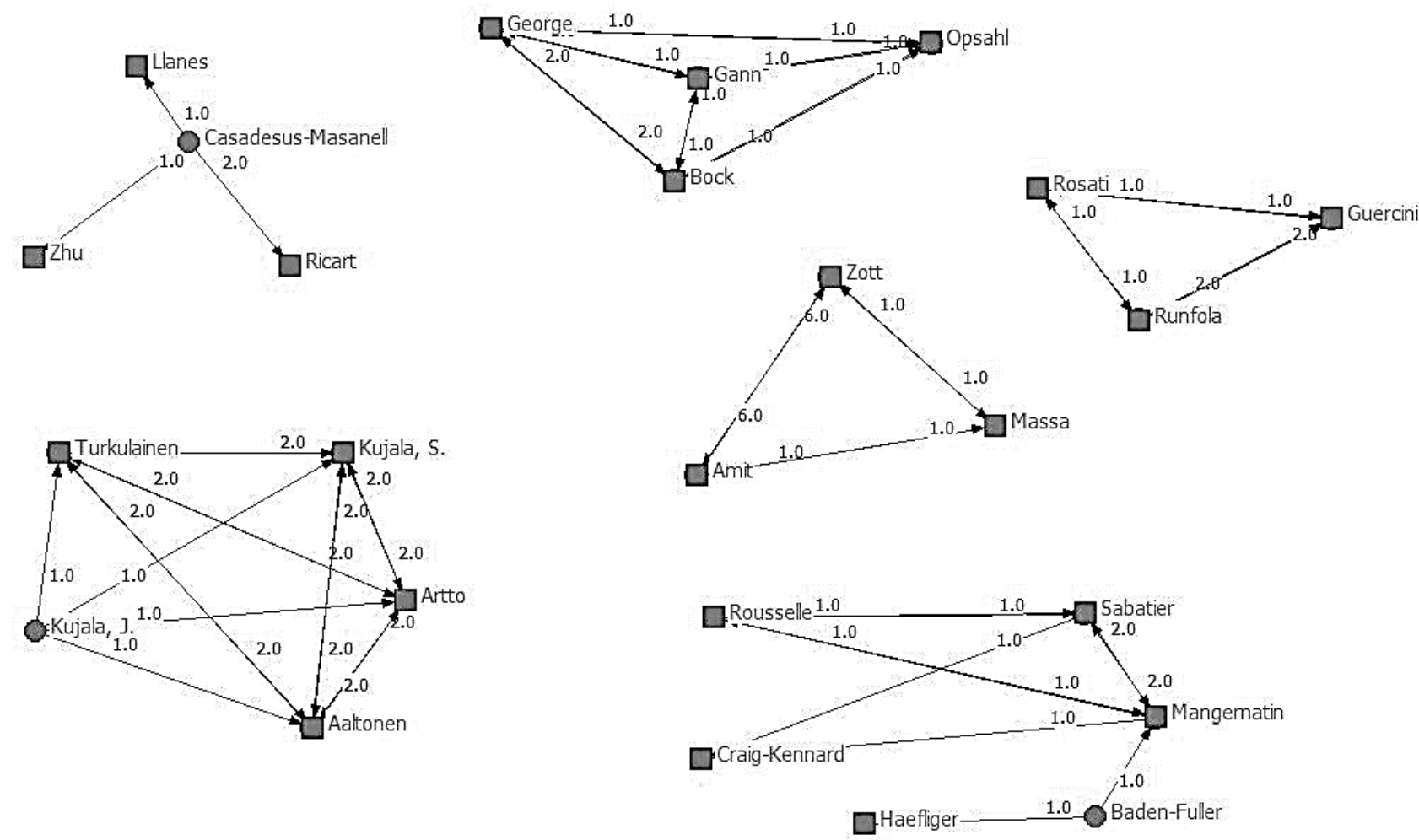

Figura 3: Rede de coautoria dos periódicos selecionados

Fonte: As autoras, segundos dados do ISI Web of Knowledge. Figura realizada com o software Ucinet. 
com duas publicações em conjunto cada um dos pares (ver Figura 3). As métricas de coautoria são importantes para indicar a dinâmica e os pontos influentes de colaboração entre os pesquisadores (THOMPSON REUTERS, 2008).

Salienta-se que Christoph Zott e Raphael Amit fizeram parte da parceria Insead - Wharton Alliance Center for Global Research and Development. Juntos, desenvolveram muitos working papers sobre o tema de Business Models voltados para os temas de estratégia empresarial e empreendedorismo. Ainda, Charles Baden-Fuller foi editor do periódico Long Range Planning durante os special issues de 2010 e 2013. Atualmente coordena um Centro de Pesquisa sobre o tema na Cass Business School (Reino Unido). Os pesquisadores Stefan Haefliger (Cass Business School) e Vicent Mangematin (Grenoble Ecole de Management) fazem parte da equipe produzindo estudos empíricos para compreender a co-evolução da tecnologia com business models em diferentes setores econômicos na Europa.

Shafer, Smith e Linder (2005) desenvolveram a

\begin{tabular}{|l|}
\hline \multicolumn{1}{|c|}{ CRIAÇÃo DE VALOR } \\
\hline -Recursos \\
- Capacidades \\
-Atividades \\
- Processos \\
- Rotinas \\
-Ativos \\
- Tecnologia \\
- Inovação
\end{tabular}

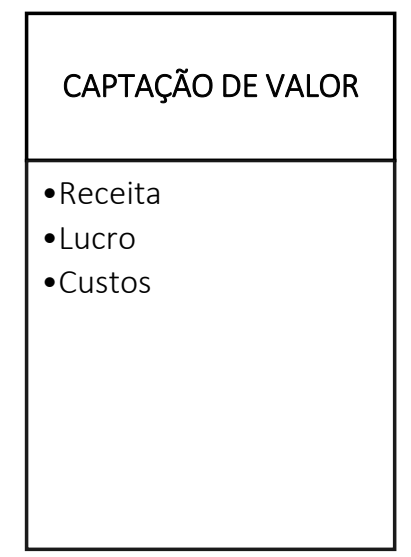

Figura 4: Componentes do business model

Fonte: As autoras

metodologia de agrupamento por afinidade do construto de business model. Com base na modelagem desenvolvida pelos autores, avaliaram-se todos os componentes citados nos estudos e estabeleceu-se uma classificação que descrevesse as ideias centrais propostas. Logo, apresenta-se abaixo, os principais componentes de business model encontrados na literatura vigente avaliada em profundidade nesta revisão.

Por criação de valor entende-se elementos internos à organização como seus ativos, competências, atividades, processos e tecnologia que auxiliam na concepção de valor ao seu cliente. Já por captura de valor, destacam-se elementos financeiros como: receita, lucro e custos da empresa, que refletem a materialização da absorção do valor entregue ao cliente.

Network está relacionada com toda a rede de relacionamento externa à empresa, ou seja, seus clientes, fornecedores, comunidade que está inserida e forma de distribuição de seus produtos e/ou serviços. Por fim, estratégia empresarial indica a própria gestão e governança da organização, com base na liderança, alinhamento de seus funcionários com metas e objetivos, a cultura organizacional e produtos e/ou serviços oferecidos.

Ao analisar os componentes, constata-se que o business model não é uma proposta de criação ou captação de valor, ou representa a integração das atividades da rede (network) da empresa. Business model é a articulação de todos esses elementos. Apesar de os autores focarem seus estudos acerca do conceito de criação de valor, a estrutura da empresa e sua governança influenciam na reconfiguração da cadeia de valor, bem como na exploração dos

\begin{tabular}{|c|c|}
\hline NETWORK & $\begin{array}{l}\text { ESTRATÉGIA } \\
\text { EMPRESARIAL }\end{array}$ \\
\hline $\begin{array}{l}\text { - Clientes } \\
\text { - Fornecedores } \\
\text { - Cadeia de } \\
\text { suprimentos } \\
\text { - Shareholders } \\
\text { - Distribuição }\end{array}$ & $\begin{array}{l}\text { - Produtos/serviços } \\
\text { - Governança/estrutura } \\
\text { - Alinhamento dos } \\
\text { funcionários } \\
\text { - Gestão } \\
\text { - Liderança } \\
\text { - Cultura }\end{array}$ \\
\hline
\end{tabular}

recursos e capacidades da empresa. Portanto, a estratégia da organização, bem como seu posicionamento e cultura são elementos centrais no design de um business model.

Embora esta pesquisa tenha sido realizada nove anos depois de Shafer et al. (2005) e os artigos analisados foram diferentes do estudo original, o resultado do agrupamento foi bastante semelhante. Diferentemente dos autores, os quais usaram o diagrama de afinidades para propor uma definição do construto, o intuito desta pesquisa foi aprofundar o conhecimento sobre os elementos que compõem um modelo de negócios para uma futura 
instrumentalização do construto em pesquisa quantitativa.

\section{Considerações finais}

A revisão sistemática permitiu compreender a definição e evolução do construto, bem como identificar os principais elementos que compõem um business model. Ainda, a articulação entre os blocos, ancorada pela estratégia da organização é relevante para o entendimento do tema. Percebe-se que os termos resultantes do agrupamento por afinidade estão bastante alinhados com os estudos de estratégia empresarial, mesmo que a pesquisa não tenha sido limitada a esta lente teórica.

Dentre as contribuições deste estudo, destaca-se a possibilidade da utilização da classificação dos componentes do business model (sintetizados na figura 3) como norteador para a formulação do instrumento de pesquisa quantitativa. Assim, questionários podem ser elaborado com base nos elementos identificados na literatura. Por outro lado, os resultados da revisão da literatura indicam a necessidade de aprofundar o conhecimento sobre as fronteiras e permeabilidades entre os componentes de um Business Model.

Acredita-se que as empresas multinacionais sejam um objeto de estudo pertinente, uma vez que elas possuem um business model complexo que pode ser distinto entre suas subsidiárias internacionais. Logo, a fim de mapear as possíveis alterações em relação ao business model da matriz, é de grande valia entender os principais elementos que compõem esse construto para a execução da pesquisa empírica.

A limitação deste estudo foi utilizar apenas uma base de pesquisa, a ISI Web of Knowledge e restringir a amostra com base no indicador de Impact Factor do JCR. Além disso, não foram avaliadas as autocitações (self-citations) entre os autores pesquisados. Contudo, percebe-se a carência de trabalhos no âmbito nacional sobre o tema, uma vez que não foram identificados autores ou instituições brasileiras na revisão. Portanto, uma agenda de pesquisa sobre o tema será pertinente para a compreensão do construto e além da construção do conhecimento sobre business model no contexto brasileiro.

Como possibilidade de pesquisa bibliométrica complementar, sugere-se a análise de citações e cocitações dos artigos do quadro 3. Ainda, indica-se a realização de estudos qualitativos para compreender elementos tácitos do business model como indicado por Baden-Fuller e Morgan (2010).

\section{Referências}

- AMIT, R.; ZOTT, C. Value creation in E-business. Strategic Management Journal, v. 22, n. 6-7, p. 493520, jun.2001. DOI: 10.1002/smj.187

- $\quad$ AREND, R. J. The business model: Present and future-beyond a skeumorph. Strategic Organization, v. 11, n. 4, p. 390-402, 8 set. 2013. DOI: $10.1177 / 1476127013499636$

- ASPARA, J.; LAMBERT, J.; LAUKIA, A.; TIKKANEN, H.Corporate Business Model Transformation and InterOrganizational Cognition: The Case of Nokia. Long Range Planning, v. 46, n. 6, p. 459-474, dez. 2013. DOI:10.1016/j.Irp.2011.06.001

- BADEN-FULLER, C.; HAEFLIGER, S. Business Models and Technological Innovation. Long Range Planning, v. 46, n. 6, p. 419-426, dez/2013. DOI:10.1016/j.Irp.2013.08.023

- BADEN-FULLER, C.; MANGEMATIN, V. Business models: A challenging agenda. Strategic Organization, v. 11, n. 4, p. 418-427, 7 nov. 2013. DOI: $10.1177 / 1476127013510112$

- BADEN-FULLER, C.; MORGAN, M. S. Business Models as Models. Long Range Planning, v. 43, n.2, p. 156-171, 2010. DOI:10.1016/j.Irp.2010.02.005

- BENIJTS, T. A Business Sustainability Model for Government Corporations. A Belgian Case Study. Business Strategy and the Environment, v. 23, n. 3, p. 204-216, mar.2014. DOI: 10.1002/bse.1784

- BOCK, A.; OPSAHL, T.; GEORGE, G.; GANN, D. The Effects of Culture and Structure on Strategic Flexibility during Business Model Innovation. Journal of Management Studies, v.49, n.2, p. 279-305, 2012. DOI: 10.1111/j.1467-6486.2011.01030.x

- BONACCORSI, A. .; GIANNANGELI, S. .; ROSSI, C. Entry Standards: Strategies Under Competing in the Open Source Business Models Hybrid Software Industry. Management science, v. 52, n. 7, p. 1085-1098, 2006. DOI: $10.1287 / \mathrm{mnsc} .1060 .0547$

- BRYSON, J. R.; LOMBARDI, R. Balancing Product and Process Sustainability against Business Profi tability: Sustainability as a Competitive Strategy in the Property Development Process. Business Strategy and the Environment, v. 107, p. 97-107, 2009. DOI: $10.1002 /$ bse. 640

- CAGNINA, M. R.; POIAN, M. Beyond e-business models: the road to virtual worlds. Electronic Commerce Research, v. 9, n. 1-2, p. 49-75, 19 mar.2009. DOI 10.1007/s10660-009-9027-3

- $\quad$ CASADESUS-MASANELL, R.; RICART, J. E. From Strategy to Business Models and onto Tactics. Long Range Planning, v. 43, n. 2-3, p. 195-215, abr.2010. DOI:10.1016/j.Irp.2010.01.004 
- CASADESUS-MASANELL, R.; RICART, J. E. How to Design A Winning Business Model. Harvard Business Review, n. January-February, p. 100-107, 2011.

- Chesbrough, H. Business Model Innovation: Opportunities and Barriers. Long Range Planning, v. 43, n. 2-3, p. 354-363, abr. 2010. DOI:10.1016/j.Irp.2009.07.010

- DAHAN, N. M. DOH, J.; OETZEL, J.; NIZIJI, M.. CorporateNGO Collaboration: Co-creating New Business Models for Developing Markets. Long Range Planning, v. 43, n. 2-3, p. 326-342, abr. 2010. DOI:10.1016/j.Irp.2009.11.003

- DASILVA, C. M.; TRKMAN, P. Business Model: What It is and What It Is Not. Long Range Planning, v. 47, n. 6 p. 379-389, 2014. DOI:10.1016/j.Irp.2013.08.004

- DAVIES, A.; BRADY, T.; HOBDAY, M. Organizing for solutions: Systems seller vs. systems integrator. Industrial Marketing Management, v. 36, n. 2, p. 183193, fev.2007. DOI:10.1016/j.indmarman.2006.04.009

- DEMIL, B.; LECOCQ, X. Business Model Evolution: In Search of Dynamic Consistency. Long Range Planning, $v$. 43, n. 2-3, p. 227-246, 2010 DOI:10.1016/j.Irp.2010.02.004

- DOgANOVA, L.; EYQUEM-RENAULT, M. What do business models do? Innovation devices in technology entrepreneurship. Research Policy, v. 38, n. 10, p. 1559-1570, dez.2009. DOI:10.1016/j.respol.2009.08.002

- DUNFORD; R.; PALMER, I.; BENVENISTE, J. Business Model Replication for Early and Rapid Internationalisation. Long Range Planning, v. 43, n. 2-3, p. 655-674, 2010. DOI:10.1016/j.Irp.2010.06.004

- ECKHARDT, J. T. Opportunities in business model research. Strategic Organization, v. 11, n. 4, p. 412-417, 7 nov.2013. DOI: 10.1177/1476127013511059

- FINK, A. Conducting research literature reviews. From the internet to paper. London: SAGE Publications, 2010.

- Gambardella, A.; MCGahan, A. M. Business-Model Innovation: General Purpose Technologies and their Implications for Industry Structure. Long Range Planning, v. 43, n. 2-3, p. 262-271, 2010. DOI:10.1016/j.Irp.2009.07.009

- GEORGE, G.; BOCK, A. J. The Business Model in Practice and its Implications for Entrepreneurship Research. Entrepreneurship Theory and Practice, v. 35, n. 1, p. 83111, 17 jan. 2011. DOI: 10.1111/j.15406520.2010.00424.x

- GIROTRA, K.; NETESSINE, S. Business Model Innovation for Sustainability Business Model Innovation for Sustainability. Working Paper, Insead. Acesso em: 10/06/2014. Disponível em: http://www.insead.edu/facultyresearch/research/doc. $\mathrm{cfm}$ ?did=52400.

- GLANZEL, W. Bibliometrics as a Research Field: A course on theory and application of bibliometric indicators.
2003. Course Handouts. Acesso em: 20/10/2014. Disponível em: http://www.cin.ufpe.br/ ajhol/futuro/references/01\% 23_Bibliometrics_Module_KUL_BIBLIOMETRICS\%20AS \%20A\%2ORESEARCH\%2OFIELD.pdf

- HUELSBECK, D. P.; MERChANT, K. A; SANDINO, T. On Testing Business Models. The Accounting Review, v. 86, n. 5, p. 1631-1654, set./2011.

- ITAMI, H.; NISHINO, K. Killing Two Birds with One Stone. Long Range Planning, v. 43, n. 2-3, p. 364-369, abr. 2010. DOI:10.1016/j.Irp.2009.07.007

- $\quad$ KEAR, R.; COLBERT-LEWIS, D. Citation searching and bibliometric measures. College \& Research Libraries. 2011. Acesso em: 20/10/2014. Disponível em: http://crln.acrl.org/content/72/8/470.full.pdf+html

- $\quad$ KLANG, D.; WALLNOFER, M.; HACKLIN, F. The Business Model Paradox: Systematic Review and Exploration of Antecendents. Interntional Journal of Management Review, v. 16, n.4, p. 454-478, 2014. DOI: 10.1111/ijmr.12030

- MAgRetTA, J. Why business models matter. Harvard Business Review, n. May, p. 1-9, 2002.

- MAHADEVAN, B. Business Models for Internet-Based ECommerce. California Management Review, v. 42, n. 4, p. $55-70,2000$

- MARKIDES, G. G. Business Model Innovation : What Can Ambidexterity Literature Teach Us. Academy of Management Perspectives, v. 27, n. 4, p. 313-323, 2013. DOI: 10.5465/amp.2012.0172

- MCNAMARA, P.; PECK, S. I.; SASSON, A. Competing Business Models, Value Creation and Appropriation in English Football. Long Range Planning, v. 46, n. 6, p. 475-487, dez./2013. DOI:10.1016/j.Irp.2011.10.002

- MUTKA, S.; AALTONEN, P. The impact of a delivery project's business model in a project-based firm. International Journal of Project Management, v. 31, n. 2, p. 166-176, fev.2013. DOI:10.1016/j.ijproman.2012.07.006

- PATZELT, H.; ZU KNYPHAUSEN-AUFSES, D.; NIKOL, P. Top Management Teams, Business Models, and Performance of Biotechnology Ventures: An Upper Echelon Perspective. British Journal of Management, v. 19, n. 3, p. 205-221, set. 2008. DOI: 10.1111/j.14678551.2007.00552.x

- RUNFOlA, A.; ROSATI, M.; GUERCINI, S. New business models in online hotel distribution: emerging private sales versus leading IDS. Service Business, v. 7, n. 2, p. 183-205, 3 jul. 2012. DOI: 10.1007/s11628-012-01501

- SABAtier, V.; CRAIG-KEnNARD, A.; MANGEMAtin, V. When technological discontinuities and disruptive business models challenge dominant industry logics: Insights from the drugs industry. Technological Forecasting and Social Change, v. 79, n. 5, p. 949-962, jun. 2012. DOI:10.1016/j.techfore.2011.12.007 
- $\quad$ SABAtier, V.; MANGEMATIN, V.; ROUSSELle, T. From Recipe to Dinner: Business Model Portfolios in the European Biopharmaceutical Industry. Long Range Planning, v. 43, n. 2-3, p. 431-447, abr. 2010. DOI:10.1016/j.Irp.2010.02.001

- $\quad$ SANTOS, J.; SPECTOR, B. ;VAN DER HEYDEN, L. Towards a theory of business model innovation within incubent firms. Working Paper. Insead, 2009.

- $\quad$ SHAFER, S.; SMITH, H; LINDER, J. The power of business model. Business Horizons, v. 48, n. 3, p. 199-207, 2005 DOI:10.1016/j.bushor.2004.10.014

- $\quad$ SMITH, W. K.; BINNS, A.; TUSHMAN, M. L. Complex Business Models: Managing Strategic Paradoxes Simultaneously. Long Range Planning, v. 43, n. 2-3, p. 448-461, abr./2010. DOI:10.1016/j.Irp.2009.12.003

- SOSNA, M.; TREVINYO-RODRIGUEZ, R.; VELAMURI, S. Business Model Innovation through Trial-and-Error Learning. Long Range Planning, v. 43, n. 2-3, p. 383-407, 2010. DOI:10.1016/j.Irp.2010.02.003

- $\quad$ STORBACKA, K. A solution business model: Capabilities and management practices for integrated solutions. Industrial Marketing Management, v. 40, n. 5, p. 699711, jul./2011. DOI:10.1016/j.indmarman.2011.05.003

- TALLMAN, S. Business Models and the Multinational Firm. In: BODDEWYN, J. Multidisciplinary Insights from New AIB Fellows (Research in Global Strategic Management, Volume 16), Emerald Group Publishing Limited, p.115-138, 2014.

- TEECE, D. J. Business Models, Business Strategy and Innovation. Long Range Planning, v. 43, n. 2-3, p. 172194, abr./2010. DOI:10.1016/j.Irp.2009.07.003

- THOMPSON REUTERS. Whitepaper Using Bibliometrics: A guide to evaluating research performance with citation data. 2008. Acesso em: 20/10/2014. Disponível em: http://thomsonreuters.com/products/ipscience/04_030/using-bibliometrics-a-guide-toevaluating-research-performance-with-citationdata.pdf

- TRIMI, S.; BERBEGAL-MIRABENT, J. Business model innovation in entrepreneurship. International Entrepreneurship and Management Journal, v. 8, n. 4, p. 449-465, 8 set./2012. DOI 10.1007/s11365-012-02343
- Willemstein, L.; VAN DER VALK, T.; MEEUS, M. T. H. Dynamics in business models: An empirical analysis of medical biotechnology firms in the Netherlands. Technovation, v. 27, n. 4, p. 221-232, 2007. DOI:10.1016/j.technovation.2006.08.005

- WINTER, S. G.; SZULANSKI, G.; HALL, H. Replication as Strategy. Organization Science, v. 12, n. 6, p. 730-743, 2001.

- WIRTZ, B. W.; SCHILKE, O.; ULLRICH, S. Strategic Development of Business Models. Long Range Planning, v. 43, n. 2-3, p. 272-290, abr./ 2010. DOI:10.1016/j.Irp.2010.01.005

- WiTELL, L.; LÖFGREN, M. From service for free to service for fee: business model innovation in manufacturing firms. Journal of Service Management, $v$. 24, n. 5, p. 520-533, 2013. DOI 10.1108/JOSM-042013-0103

- Yunus, M.; MOINGEON, B.; LEHMANN-ORTEGA, L. Building Social Business Models: Lessons from the Grameen Experience. Long Range Planning, v. 43, n. 23, p. 308-325, abr./2010. DOI:10.1016/j.Irp.2009.12.005

- ZOTT, C.; AMIT, R. Business Model Design and the Performance of Entrepreneurial Firms. Organization Science, v.18, n.2, p.181-199, 2007. http://dx.doi.org/10.1287/orsc.1060.0232

- ZOTT, C.; AMIT, R. The Fit Between Product Market Strategy And Business Model: Implications For Firm Performance. Strategic Management Journal, v. 29, n.1, p. 1-26, 2008. DOI: 10.1002/smj.642

- ZOTT, C.; AMIT, R. Business Model Design: An Activity System Perspective. Long Range Planning, v. 43, n. 2-3, p. 216-226, abr./2010. DOI:10.1016/j.Irp.2009.07.004

- ZOTT, C.; AMIT, R. The business model: A theoretically anchored robust construct for strategic analysis. Strategic Organization, v. 11, n. 4, p. 403-411, 7 nov. 2013. DOI: $10.1177 / 1476127013510466$

- ZOTT, C.; AMIT, R.; MASSA, L. The Business Model: Recent Developments and Future Research. Journal of Management, v. 37, n. 4, p. 1019-1042, mai./2011. DOI: $10.1177 / 0149206311406265$

\section{SOBRE AS AUTORAS}

- Cyntia Vilasboas Calixto é doutoranda em Administração de Empresas pela Escola de Administração da Fundação Getúlio - EAESP/FGV, São Paulo/SP, Brasil. Email: cyntiacalixto@gmail.com

- Maria Tereza Leme Fleury é doutora em Administração de Empresas pela Universidade de São Paulo (USP) e atualmente é professora de Estratégia Internacional na Escola de Administração de Empresas da Fundação Getúlio Vargas - EAESP/FGV, São Paulo/SP, Brasil. Email: mtereza.fleury@fgv.br 


\title{
Business model: Unveiling the construct
}

\author{
Cyntia Vilasboas Calixto and Maria Tereza Leme Fleury
}

Fundação Getúlio Vargas - EAESP/FGV, São Paulo/SP, Brazil

\section{ARTICLE DETAILS}

\section{Article history:}

Received 31 October 2014

Accepted 12 June 2015

Available online in 30 August 2015

Double Blind Review System

\section{Scientific Editors}

Gabriel Vouga Chueke

Marcos Amatucci

\section{Keywords:}

Business model

Multinational companies

International business

\begin{abstract}
This essay was developed based on a systematic literature review to identify the main definitions of business model as well as the elements that compose this construct. We analyzed 81 papers published in journals with scores above 1.5 according to Journal Citation Report (JCR) standards. We realized that the relationship between business model and multinational enterprises has been neglected by researchers and therefore appears as an opportunity for research. Considering that business models describe how a company creates value through a combination of internal and external resources within their operations, it is important to understand its design elements to determine a business model for multinational enterprises
\end{abstract}

